\title{
On Saint-Venant's principle in plane anisotropic elasticity
}

\author{
CORNELIUS O. HORGAN ${ }^{1}$ ) \\ Department of Engineering Mechanics, The University of Michigan, Ann Arbor, Michigan.
}

(Received July 15, 1972)

\begin{abstract}
Methods involving en ergy-decay inequalities are applied in investigating Saint-Venant's principle for the planproblem of linear elastostatics for a wide class of anisotropic media. A lower bound (in terms of the elastic constants) is obtained for the rate of exponential decay of stresses and this is compared with the known result for the isotropic case.

\section{RÉSUMÉ}

Par une méthode applicable à un très grand nombre de milieu anisotropique, l'auteur utilise les inégalités concernant la décroissance de l'énergie dans le cas d'un problème plan et dans l'hypothèse de conditions élastostatiques linéaires, l'auteur précise la validité du principe de Saint-Venant dans le cadre de ses applications. Une limite in férieure (en fonction des constantes élastiques) de la décroissance exponentielle des contraintes est mise en évidence et comparée aux résultats obtenus en milieux isotropes.
\end{abstract}

\section{Introduction}

In recent years, several attempts at clarification of Saint-Venant's principle in elasticity theory have been made. In essence the work reported in [1-4] has been concerned with establishing the exponential decay of strain energy away from that portion of the boundary of an elastic solid which is subjected to a self-equilibrated load. This energy decay inequality in turn leads to pointwise estimates for the stresses at interior points. The restriction of this result to strictly interior points was removed by Roseman [5], who also considered the effect of self-equilibrated loads in nonlinear elasticity [6].

Saint-Venant's principle for second order elliptic boundary value problems was considered by Knowles in [7] (see also Wu [8]), while more recently, Edelstein has investigated the issue for linear viscoelasticity [9] and for a general class of parabolic equations [10], [11]. See also Sigillito [12] and Knowles [13]. References to earlier contributions may be found in [1-6].

The present work is concerned with the two-dimensional problem of linear elastostatics for a wide class of anisotropic media. The analysis is similar to that of Knowles [2] for the isotropic case. A comparison between the decay constant obtained here (which provides a lower bound for the actual rate of decay) with that obtained in [2] suggesis that the "characteristic decay length" associated with Saint-Venant's principle is smallest in the isotropic case. The result indicates that care must be exercised in predicting the extent of "end effects" in anisotropic solids.

In the following section, we describe the traction boundary value problem of plane strain for an homogeneous anisotropic cylindrical body with one plane of elastic symmetry, while in Section 3 we formulate the issue associated with Saint-Venant's principle. The derivation of the exponential decay inequality is contained in Section 4, which concludes with a brief dis-

${ }^{1}$ ) Now at School of Mathematics and Physics, University of East Anglia, Norwich, England. 
cussion of pointwise estimates for the stresses at interior points. In the last section we discuss the decay constant, with particular emphasis on comparison with the result for the isotropic case.

Finally here we note that the formulation of Saint-Venant's principle as given by Sternberg in [14] also applies to an anisotropic medium (see the comments in [15]), as does the analysis of Toupin in [1].

\section{Plane deformation of an anisotropic solid}

We wish to consider the plane elastostatic problem associated with the equilibrium of an homogeneous elastic cylindrical body, with simply connected cross section, under the action of surface tractions which act in planes normal to the generators and do not vary along them. Let $x_{1}$, $x_{2}$ be rectangular Cartesian coordinates in a fixed cross section and denote the interior and boundary of this cross section by $R$ and $C$ respectively, where $C$ is assumed to be a piecewise smooth closed curve.

As is discussed in Section 21 of Lekhnitskii [16], a general anisotropic solid under such conditions does not give rise to a state of plane deformation. However, for a body with the $x_{1}, x_{2}$ plane a plane of elastic symmetry, then it is possible, (see Section 22 of [16]) to define a state of "pure plane deformation", henceforth called plane strain, such that

$$
u_{\alpha}=u_{\alpha}\left(x_{1}, x_{2}\right), u_{3}=0 \text {, }
$$

where $u_{i}$ denotes the Cartesian components of the displacement vector $\boldsymbol{u} .^{1}$ ) The components of the strain tensor are given by

$$
e_{i j}=\frac{1}{2}\left(u_{i, j}+u_{j, i}\right) \text {. }
$$

The stress-strain relations for a material with one plane of elastic symmetry (the $x_{1}, x_{2}$ plane) are given in Section 3 of [16] as follows

$$
\left.\begin{array}{rl}
e_{11} & =a_{11} \tau_{11}+a_{12} \tau_{22}+a_{13} \tau_{33}+a_{16} \tau_{12} \\
e_{22} & =a_{21} \tau_{11}+a_{22} \tau_{22}+a_{23} \tau_{33}+a_{26} \tau_{12} \\
e_{33} & =a_{31} \tau_{11}+a_{32} \tau_{22}+a_{33} \tau_{33}+a_{36} \tau_{12} \\
2 e_{23} & =a_{44} \tau_{23}+a_{45} \tau_{31} \\
2 e_{31} & =a_{54} \tau_{23}+a_{55} \tau_{31} \\
2 e_{12} & =a_{61} \tau_{11}+a_{62} \tau_{22}+a_{63} \tau_{33}+a_{66} \tau_{12}
\end{array}\right\},
$$

where $a_{p q}=a_{q p}(p, q=1,2, \ldots 6)$ are the elastic compliances (called the coefficients of deformation in [16]) and $\tau_{i j}=\tau_{j i}$ denote the components of the stress tensor; there are at most 13 independent constants in (2.3). Using the plane strain condition (2.1), we may write the nonzero strains $e_{\alpha \beta}\left(x_{1}, x_{2}\right)$ as

$$
\left.\begin{array}{rl}
e_{11} & =\beta_{11} \tau_{11}+\beta_{12} \tau_{22}+\beta_{16} \tau_{12} \\
e_{22} & =\beta_{21} \tau_{11}+\beta_{22} \tau_{22}+\beta_{26} \tau_{12} \\
2 e_{12} & =\beta_{61} \tau_{11}+\beta_{62} \tau_{22}+\beta_{66} \tau_{12}
\end{array}\right\} .
$$

The constants $\beta_{p q}=\beta_{q p}$ are given by

1) Cartesian tensor notation is used in Sections 2, 3 of this paper, with summation over repeated subscripts implied. Greek and Latin subscripts have the range 1,2 and 1,2,3 respectively, unless specifically stated otherwise. 


$$
\beta_{p q}=a_{p q}-\frac{a_{p 3} a_{q 3}}{a_{33}}(p, q=1,2,6)
$$

where it is assumed that $a_{33} \neq 0$.

In the absence of body forces, the stresses of primary interest $\tau_{\alpha \beta}\left(x_{1}, x_{2}\right)$ must satisfy the equilibrium equations

$$
\tau_{\alpha \beta, \beta}=0 \text { in } R,
$$

subject to appropriate boundary conditions on $C$. We now introduce the Airy stress function $\phi$ by

$$
\tau_{\alpha \beta}=\varepsilon_{\alpha \lambda} \varepsilon_{\beta \mu} \phi, \lambda \mu,
$$

where $\varepsilon_{\alpha \beta}$ denotes the two-dimensional alternator $\varepsilon_{11}=\varepsilon_{22}=0, \varepsilon_{12}=-\varepsilon_{21}=1$. Then (2.6) are identically satisfied and the relevant strain compatibility equation, on using (2.4), (2.7) yields the fourth order partial differential equation ${ }^{1}$ )

$$
\beta_{22} \phi_{, 1111}-2 \beta_{26} \phi_{, 1112}+\left(2 \beta_{12}+\beta_{66}\right) \phi_{, 1122}-2 \beta_{16} \phi_{, 1222}+\beta_{11} \phi_{, 2222}=0 \text { in } R \text {. }
$$

The boundary conditions to be satisfied by $\phi$ may be written as

$$
\varepsilon_{\alpha \lambda} \varepsilon_{\beta \mu} \phi, \lambda \mu n_{\beta}=T_{\alpha} \text { on } C,
$$

where $T_{\alpha}\left(x_{1}, x_{2}\right)$ is the $x_{\alpha}$-component of the surface traction vector $T$ given on $C$ and $n_{\alpha}$ denotes the $x_{\alpha}$-component of the unit outward normal vector $n$ on $C$. The surface tractions $\boldsymbol{T}$ must be such that overall equilibrium holds.

Finally here we note that equations (2.6)-(2.9) also apply in the case of generalized plane stress, where $\tau_{\alpha \beta}, T_{\alpha}$ are the averages over the cylinder thickness and $\beta_{p q}$ are replaced by $a_{p q}$. (See Section 22 of [16].)

\section{Formulation of the boundary value problem}

To investigate the issue of Saint-Venant's principle in the present context, it is sufficient to consider the effect of a surface traction $\boldsymbol{T}$ which is self-equilibrated on a portion of the domain boundary $C$. Thus, we suppose, following [2], that the coordinate axes are arranged as shown

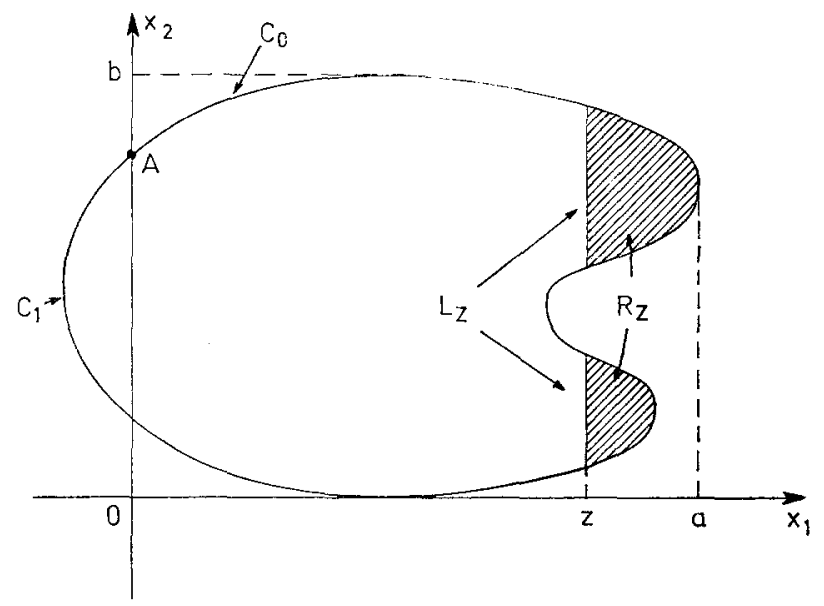

Fig. 1

1) Cr. equation (22.5) of [16]. 
in Fig. 1, where $C_{1}$ is the loaded arc and $C_{0}$ is traction free. We also use the notation introduced in [2], namely

$$
\begin{aligned}
& a=\max _{C_{0}} x_{1}, \quad b=\max _{c_{0}} x_{2}, \\
& R_{z}=\left\{\left(x_{1}, x_{2}\right) \mid\left(x_{1}, x_{2}\right) \in R, x_{1}>z\right\}, \\
& L_{z}=\left\{\left(x_{1}, x_{2}\right) \mid\left(x_{1}, x_{2}\right) \in R, x_{1}=z\right\},
\end{aligned}
$$

and we let $\mathscr{B}\left(R_{z}\right)$ denote the set of functions $f \in C^{2}\left(\bar{R}_{z}\right), C^{4}\left(R_{z}\right)$, with bounded third derivatives on $R_{z} ; \mathscr{B}(R)$ denotes the class of functions with these properties on $R$. It is shown in [2] that the boundary conditions (2.9) can be integrated to give

$$
\begin{aligned}
& \phi=\left\{\begin{array}{ll}
\int_{0}^{s} \varepsilon_{\alpha \beta}\left[x_{\beta}(s)-x_{\beta}(\sigma)\right] T_{\alpha}(\sigma) d \sigma & \text { on } C_{1}, \\
0 & \text { on } C_{0},
\end{array}\right\} \\
& \phi,{ }_{\alpha}=\left\{\begin{array}{ll}
\int_{0}^{s} \varepsilon_{\beta \alpha} T_{\beta}(\sigma) d \sigma & \text { on } C_{1}, \\
0 & \text { on } C_{0}
\end{array}\right\}
\end{aligned}
$$

where $s$ denotes arc length on $C$ measured counter-clockwise from $A$ in Fig. 1 . The fact that $\phi$ and its first partial derivatives vanish at the end points of $C_{1}$ is a consequence of the self-equilibration conditions on $\boldsymbol{T}$. (See [2].) Our main result in Section 4 will establish the exponential decay with $z$ of the strain energy $E(z)$ contained in the subdomain $R_{z}$.

We conclude this section with some preliminary remarks which will be of use in the sequel. The total strain energy associated with the boundary value problem considered here is $\int_{R} \int W \mathrm{~d} A$, where the strain-energy density $W$ is given, with the aid of (2.4) by

$$
2 W \equiv \tau_{\alpha \beta} e_{\alpha \beta}=\beta_{11} \tau_{11}^{2}+\beta_{22} \tau_{22}^{2}+\beta_{66} \tau_{12}^{2}+2 \beta_{12} \tau_{11} \tau_{22}+2 \beta_{16} \tau_{11} \tau_{12}+2 \beta_{26} \tau_{22} \tau_{12} .
$$

We assume henceforth that $W(\tau)$ is positive definite throughout $R$, that is $W(\tau)>0$ for all $\tau \neq 0$. I $\mathrm{t}$ is convenient here to introduce the matrix notation $2 W=t^{\prime} B t$, where $t$ is the column vector

$$
t=\left[\begin{array}{l}
\tau_{11} \\
\tau_{22} \\
\tau_{12} \sqrt{2}
\end{array}\right],
$$

$t^{\prime}$ is the transpose of $t$ and the symmetric matrix $B$ is

$$
B=\left[\begin{array}{lll}
\beta_{11} & \beta_{12} & \frac{\beta_{16}}{\sqrt{2}} \\
\beta_{12} & \beta_{22} & \frac{\beta_{26}}{\sqrt{2}} \\
\frac{\beta_{16}}{\sqrt{2}} & \frac{\beta_{26}}{\sqrt{2}} & \frac{\beta_{66}}{2}
\end{array}\right] .
$$

The norm of the stress tensor is $|\tau|=\left(\tau_{\alpha \beta} \tau_{\alpha \beta}\right)^{\frac{1}{2}}=\left(t^{\prime} t\right)^{\frac{1}{2}}$. We note that $W$ is positive definite if and only if all the eigenvalues of $B$ are positive. Furthermore, it readily follows from the standard diagonalization of real symmetric matrices that $W$ satisfies

$$
2 W \geqq \lambda|\tau|^{2},
$$

where $\lambda>0$ is the minimum eigenvalue of $B$ and is optimal in (3.5). 
Finally here, we recall a result derived in [2] concerning the differentiation of integrals over $R_{z}$.

Thus

$$
\frac{d}{d z} \int_{R_{z}} \int f d A=-\int_{L_{z}} f d x_{2}
$$

for all $R_{z} \subset R$, where $f$ is continuous on $\bar{R}$. This formula is an immediate consequence of the definition of the derivative on the left in (3.6).

\section{Energy decay estimates}

Before proceeding to the proof of our main theorem, it is convenient to change notation and use $x, y$ instead of $x_{1}, x_{2}$ respectively. Also we write $\phi_{x}, \phi_{y}$ in place of $\phi_{, \alpha}$, with corresponding notation for the higher parial derivatives. The energy $E(z)$, on using (2.7) and (3.3) is given by

$$
\begin{aligned}
2 E(z)= & \int_{R_{z}} \int 2 W d A=\int_{R_{z}} \int\left(\beta_{11} \phi_{y y}^{2}+\beta_{22} \phi_{x x}^{2}\right. \\
& \left.+\beta_{66} \phi_{x y}^{2}+2 \beta_{12} \phi_{x x} \phi_{y y}-2 \beta_{16} \phi_{y y} \phi_{x y}-2 \beta_{26} \phi_{x x} \phi_{x y}\right) d A .
\end{aligned}
$$

THEOREM 1. Let $\phi \in \mathscr{B}\left(R_{0}\right)$ and suppose that

$$
\beta_{22} \phi_{x x x x}-2 \beta_{26} \phi_{x x x y}+\left(2 \beta_{12}+\beta_{66}\right) \phi_{x x y y}-2 \beta_{16} \phi_{x y y y}+\beta_{11} \phi_{y y y y}=0 \text { on } R_{0} \text {, }
$$

subject to

$$
\phi=\phi_{x}=\phi_{y}=0 \text { on } C_{0} .
$$

Then for $0 \leqq z \leqq a$,

$$
E(z) \leqq 2 E(0) e^{-2 k z}
$$

where the constant $k$ is the positive root of equation (4.22).

Proof. The argument that follows closely parallels that given in the proof of Theorem 1 of [2]. Thus our aim is to establish a differential inequality which relates $E(z)$ and the integral $\int_{z}^{a} E(x) d x$. To begin with, we derive a convenient line integral representation for this integral.

For $0<z<a$, we rewrite (4.1), on using (4.2), in the form

$$
\begin{aligned}
2 E(z)= & \int_{R_{z}} \int\left[\frac { \partial } { \partial x } \left\{\beta_{22}\left(\phi_{x} \phi_{x x}-\phi \phi_{x x x}\right)+\beta_{66} \phi_{y} \phi_{x y}\right.\right. \\
& \left.+2 \beta_{12} \phi_{x} \phi_{y y}-2 \beta_{12} \phi \phi_{y y x}-2 \beta_{16} \phi_{y y} \phi_{y}-2 \beta_{26} \phi_{x x} \phi_{y}\right\} \\
& \left.+\frac{\partial}{\partial y}\left\{\beta_{11}\left(\phi_{y} \phi_{y y}-\phi \phi_{y y y}\right)-\beta_{66} \phi \phi_{x x y}+2 \beta_{16} \phi \phi_{x y y}+2 \beta_{26} \phi \phi_{x x x}\right\}\right] d A .
\end{aligned}
$$

Application of Green's theorem and the boundary conditions (4.3) yield the representation

$$
\begin{aligned}
2 E(z)= & -\int_{L_{z}} h d y \\
= & -\int_{L_{z}}\left[\beta_{22}\left(\phi_{x} \phi_{x x}-\phi \phi_{x x x}\right)+\beta_{66} \phi_{y} \phi_{x y}+2 \beta_{12}\left(\phi_{x} \phi_{y y}-\phi \phi_{y y x}\right)\right. \\
& \left.-2 \beta_{16} \phi_{y y} \phi_{y}-2 \beta_{26} \phi_{x x} \phi_{y}\right] d y,
\end{aligned}
$$


for $0<z<a$. Since $\phi \in \mathscr{B}\left(R_{0}\right)$ we may use (3.6) to write

$$
2 E(z)=\frac{d}{d z} \int_{R_{z}} \int h d A,
$$

so that integration from $z$ to $a$ gives

$$
\int_{z}^{a} 2 E(x) d x=-\int_{R_{z}} \int h d A,
$$

where we have made use of the fact that the integral on the right vanishes when $z=a$. We again transform (4.6) by using Green's theorem and the boundary conditions (4.3) to obtain the required representation

$$
\begin{aligned}
\int_{z}^{a} 2 E(x) d x= & -\int_{R_{z}} \int\left[\frac{\partial}{\partial x}\left\{\beta_{22}\left(\phi_{x}^{2}-\phi \phi_{x x}\right)+\frac{\beta_{66}}{2} \phi_{y}^{2}-2 \beta_{26} \phi_{x} \phi_{y}\right\}\right. \\
& \left.+\frac{\partial}{\partial y}\left\{-\beta_{16} \phi_{y}^{2}+2 \beta_{12}\left(\phi_{x} \phi_{y}-\phi \phi_{x y}\right)+\beta_{26} \phi_{x}^{2}\right\}\right] d A \\
= & \int_{L_{x}}\left[\beta_{22}\left(\phi_{x}^{2}-\phi \phi_{x x}\right)+\frac{\beta_{66}}{2} \phi_{y}^{2}-2 \beta_{26} \phi_{x} \phi_{y}\right] d y .
\end{aligned}
$$

Using (3.6) and (4.1), we write the derivative $E^{\prime}(z)$ as

$$
2 E^{\prime}(z)=-\int_{L_{z}} 2 W d y, \quad 0<z<a,
$$

where $2 W$ is given by (4.1). We introduce now the auxiliary function $F$ by setting

$$
F(z)=2 E(z)+2 k \int_{z}^{a} 2 E(x) d x, 0 \leqq z \leqq a,
$$

where $k$ is any real constant. Then (4.7), (4.8) may be combined to yield the following identity. For $0<z<a$,

$$
\begin{aligned}
F^{\prime}(z)+2 k F(z) & =2 E^{\prime}(z)+4 k^{2} \int_{z}^{a} 2 E(x) d x \\
& =-\int_{L_{z}}\left[2 W-4 k^{2}\left(\beta_{22} \phi_{x}^{2}-\beta_{22} \phi \phi_{x x}+\frac{\beta_{66}}{2} \phi_{y}^{2}-2 \beta_{26} \phi_{x} \phi_{y}\right)\right] d y .
\end{aligned}
$$

Determination of a positive value of $k$ for which the right hand side of (4.10) is nonpositive will enable us to complete the derivation of (4.4).

The remainder of the proof will be based on the inequality (3.5) and an inequality of Wirtinger type which was used by Knowles in [2]. For $\psi$ continuously differentiable with respect to $y$ on $\bar{L}_{z}$ and $\psi$ vanishing at the end points of all the line segments which constitute $L_{z}$, we have

$$
\int_{L_{z}} \psi_{y}^{2} d y \geqq \frac{\pi^{2}}{b^{2}} \int_{L_{z}} \psi^{2} d y .
$$

The result (4.11) follows immediately from the basic inequality

$$
\int_{c}^{d} f_{y}(y)^{2} d y \geqq \frac{\pi^{2}}{(d-c)^{2}} \int_{c}^{d} f(y)^{2} d y
$$


for $\left.f \in C^{1}[c, d], f(c)=f(d)=0 .^{1}\right)$

Application of (3.5) in (4.10) gives

$$
\begin{aligned}
F^{\prime}(z)+2 k F(z) & \leqq-\int_{L_{z}}\left[\lambda\left(\phi_{x x}^{2}+\phi_{y y}^{2}+2 \phi_{x y}^{2}\right)\right. \\
& \left.-4 k^{2}\left(\beta_{22} \phi_{x}^{2}-\beta_{22} \phi \phi_{x x}+\frac{\beta_{66}}{2} \phi_{y}^{2}-2 \beta_{26} \phi_{x} \phi_{y}\right)\right] d y \\
& =-\int_{L_{z}}\left[\lambda\left(\phi_{x x}+\frac{2}{\lambda} k^{2} \beta_{22} \phi\right)^{2}+\lambda \phi_{y y}^{2}+2 \lambda \phi_{x y}^{2}\right. \\
& \left.-2 k^{2} \beta_{66} \phi_{y}^{2}+8 k^{2} \beta_{26} \phi_{x} \phi_{y}-4 k^{2} \beta_{22} \phi_{x}^{2}-\frac{4 k^{4}}{\lambda} \beta_{22}^{2} \phi^{2}\right] d y
\end{aligned}
$$

where $\lambda>0$ is the minimum eigenvalue of the matrix $B$ in (3.4). In view of the continuity assumptions on $\phi$ and the fact that $\phi=\phi_{x}=\phi_{y}=0$ on $C_{0}$, we readily obtain from (4.11) the inequalities

$$
\begin{aligned}
& \int_{L_{z}} \phi_{x y}^{2} d y \geqq \frac{\pi^{2}}{b^{2}} \int_{L_{z}} \phi_{x}^{2} d y, \\
& \int_{L_{z}} \phi_{y y}^{2} d y \geqq \frac{\pi^{2}}{b^{2}} \int_{L_{z}} \phi_{y}^{2} d y, \\
& \int_{L_{z}} \phi_{y}^{2} d y \geqq \frac{\pi^{2}}{b^{2}} \int_{L_{z}} \phi^{2} d y .
\end{aligned}
$$

Applying (4.14), (4.15) to (4.13) we find that

$$
\begin{aligned}
F^{\prime}(z)+2 k F(z) & \leqq-\int_{L_{z}}\left[\left(2 \lambda \frac{\pi^{2}}{b^{2}}-4 k^{2} \beta_{22}\right) \phi_{x}^{2}\right. \\
& \left.+\left(\lambda \frac{\pi^{2}}{b^{2}}-2 k^{2} \beta_{66}\right) \phi_{y}^{2}+8 k^{2} \beta_{26} \phi_{x} \phi_{y}-\frac{4}{\lambda} k^{4} \beta_{22}^{2} \phi^{2}\right] d y .
\end{aligned}
$$

The first three terms in the last integrand may be written as a quadratic form in the quantities $\phi_{x}, \phi_{y}$ as follows. Let $Q=d^{\prime} D d$ where $D$ is the symmetric matrix

$$
D=\left[\begin{array}{cc}
2 \lambda \frac{\pi^{2}}{b^{2}}-4 k^{2} \beta_{22} & 4 k^{2} \beta_{26} \\
4 k^{2} \beta_{26} & \lambda \frac{\pi^{2}}{b^{2}}-2 k^{2} \beta_{66}
\end{array}\right],
$$

and $d$ is the column vector $d=\left[\begin{array}{l}\phi_{x} x \\ \phi_{y}\end{array}\right]$. We now suppose that the undetermined constant $k$ is to be chosen such that the eigenvalues of $D$ are positive. ${ }^{2}$ ) Then since $D$ is real symmetric, we obtain the inequality

$$
Q \geqq \omega\left(d^{\prime} d\right)=\omega\left(\phi_{x}^{2}+\phi_{y}^{2}\right),
$$

where $\omega>0$ is the minimum eigenvalue of $D$. We now use (4.19) in (4.17) to get

$\left.{ }^{1}\right)$ For a direct proof of (4.12), see [17]. A variational approach to inequalities of this type may be found in [18], [19].

$\left.{ }^{2}\right) D$ positive definite is a necessary and sufficient condition for this to hold. See the discussion preceding (3.5). 


$$
F^{\prime}(z)+2 k F(z) \leqq-\int_{L_{x}}\left[\omega\left(\phi_{x}^{2}+\phi_{y}^{2}\right)-\frac{4}{\lambda} k^{4} \beta_{22}^{2} \phi^{2}\right] d y
$$

Then, since $\omega>0$ and inequality (4.16) holds, we deduce from (4.20) that

$$
F^{\prime}(z)+2 k F(z) \leqq-\left(\omega \frac{\pi^{2}}{b^{2}}-\frac{4}{\lambda} k^{4} \beta_{22}^{2}\right) \int_{L_{z}} \phi^{2} d y .
$$

The constant $k$ is now chosen to be the positive root of the equation ${ }^{1}$ )

$$
\omega \frac{\pi^{2}}{b^{2}}-\frac{4}{\lambda} k^{4} \beta_{22}^{2}=0 \text {. }
$$

With this choice of $k$, we conclude from (4.21) that $F(z)$ satisfies the differential inequality

$$
F^{\prime}(z)+2 k F(z) \leqq 0, \quad 0<z<a \text {. }
$$

The final part of the proof consists in showing that (4.23) implies (4.4), and follows exactly the argument used in [2]. Thus we see that (4.23) yields the inequality.

$$
F(z) \leqq F(0) e^{-2 k z}, \quad 0 \leqq z \leqq a .
$$

Recalling the definition (4.9) of $F(z)$ it follows from (4.24) that

$$
2 E(z) \leqq F(0) e^{-2 k z} \text {, }
$$

and so it remains to derive an upper bound for $F(0)$. Upon using (4.9) in (4.24), it is found that

$$
-\frac{d}{d z}\left[e^{-2 k z} \int_{z}^{a} 2 E(x) d x\right] \leqq F(0) e^{-4 k z} .
$$

Integration from $z=0$ to $z=a$ yields

$$
\int_{0}^{a} 2 E(x) d x \leqq \frac{F(0)}{4 k}\left(1-e^{-4 k a}\right)=\frac{1}{4 k}\left[2 E(0)+2 k \int_{0}^{a} 2 E(x) d x\right]\left(1-e^{-4 k a}\right) .
$$

When this inequality is solved for the integral of $E$ it is found that

$$
2 k \int_{0}^{a} 2 E(x) d x \leqq \frac{1-e^{-4 k a}}{1+e^{-4 k a}} 2 E(0) \leqq 2 E(0) .
$$

Together (4.27) and (4.9) show that $F(0) \leqq 4 E(0)$, so that (4.25) furnishes

$$
E(z) \leqq 2 E(0) e^{-2 k z}, \quad 0 \leqq z \leqq a,
$$

which is precisely (4.4). This completes the proof of the theorem.

The total energy $E(0)$ appearing in (4.28) can be estimated by application of an appropriate minimum principle (cf. [2], [3]) but we will not pursue this here.

We conclude this section with some remarks concerning pointwise estimates for the stresses at interior points of the domain. In [1-4] such estimates were obtained by using various mean value theorems of the theory of elasticity. In particular, for the plane problem considered in [2], a mean value theorem for biharmonic functions was used. For the fourth order equation (4.2)

$\left.{ }^{1}\right)$ Note that since $\lambda>0$ this choice of $k$ is consistent with our earlier assumption that $\omega>0$. The fact that (4.22) has just one positive root will be shown in Section 5 . 
being investigated here, we may employ a mean value theorem due to Pini $\left.[20]^{1}\right)$, together with the inequality (4.28), to establish the exponential decay of stresses (with exponent $k$ ) away from the loaded arc $C_{1}$. We note that such an estimate, in common with similar inequalities based on mean value theorems, breaks down for boundary points and is poor in the neighborhood of the boundary. It may be possible to use an alternative approach similar to that of Roseman [5], [6] to remedy this defect.

Our main interest here is in investigating the decay constant $k$, which provides a lower bound on the actual rate of exponential decay and it is to this topic that we turn next.

\section{The decay constant}

We recall that the constant $k$ is determined as the positive root of equation (4.22) where $\omega>0$ is the minimum eigenvalue of the matrix $D$ in (4.18) and $\lambda>0$ is the minimum eigenvalue of the matrix $B$ in (3.4). For a given material $B$ is known and $\lambda$ can be found, by numerical means if necessary. For our purposes here, we use an upper bound for $\lambda$ which will be described later in the section.

To simplify the following discussion, we introduce the new notation

$$
\begin{aligned}
& k^{2}=\lambda \frac{\pi^{2}}{2 b^{2}} \frac{s}{\beta_{22}}, \\
& \alpha=\frac{\beta_{66}}{2 \beta_{22}}, \quad \eta=\frac{\beta_{26}}{\beta_{22}} .
\end{aligned}
$$

Then the matrix $D$ in (4.18) may be written

$$
D=\frac{2 \lambda \pi^{2}}{b^{2}}\left[\begin{array}{cc}
1-s & \eta s \\
\eta s & \frac{1}{2}-\alpha s
\end{array}\right] .
$$

Thus we see that to determine $k$ from (4.22) we must find the positive root of the equation

$$
s^{2}=2 v,
$$

where

$$
v=\frac{b^{2} \omega}{2 \lambda \pi^{2}}>0
$$

is the minimum eigenvalue of the matrix

$$
E=\left[\begin{array}{cc}
1-s & \eta s \\
\eta s & \frac{1}{2}-\alpha s
\end{array}\right] .
$$

Consequently we seek the positive root of

$$
s^{2}=\frac{3}{2}-(1+\alpha) s-\left[\left\{\frac{1}{2}+(\alpha-1) s\right\}^{2}+4 \eta^{2} s^{2}\right]^{\frac{1}{2}}
$$

or equivalently, the smallest positive root of the quartic equation

$$
f(s) \equiv s^{4}+2(1+\alpha) s^{3}+\left(4 \alpha-3-4 \eta^{2}\right) s^{2}-2(1+2 \alpha) s+2=0 .
$$

1) For a discussion of mean value theorems for elliptic operators along the lines of Pini's work, see $\S 29$ of Miranda [21].

$\left.{ }^{2}\right)$ Note that the positive-definiteness of $B$ implies that $\beta_{11}>0, \beta_{22}>0, \beta_{66}>0$ and so $\alpha>0$. 
In the analysis that follows it is convenient to distinguish between the cases when $\beta_{26}=0$ (Case 1 ) and when $\beta_{26} \neq 0$ (Case 2). In Case $1, \eta=0$ and equation (5.7) can readily be solved explicitly with roots $-1 \pm \sqrt{3},-\alpha \pm\left(\alpha^{2}+1\right)^{\frac{1}{2}}$, of which two are negative and two positive. Denoting the smallest positive root by $s_{1}$, we then obtain the results

$$
\begin{aligned}
& s_{1}=\sqrt{3}-1 \text { if } \alpha \leqq \frac{3-\sqrt{3}}{4}, \\
& s_{1}=\left(\alpha^{2}+1\right)^{\frac{1}{2}}-\alpha \text { if } \alpha \geqq \frac{3-\sqrt{3}}{4} .
\end{aligned}
$$

In Case 2, $\eta \neq 0$ and since $\alpha>0$, Descartes' rule of signs ${ }^{1}$ ) applied to (5.7) predicts either two positive and two negative roots or all the roots complex. Noting that $f(s)$ changes sign between zero and $s_{1}$ we infer that the former holds and in addition we obtain

$$
s_{2}<s_{1}
$$

where $s_{2}$ is the smallest positive root of (5.7) in Case 2. ${ }^{2}$ )

We return now to the characterization of the decay constant $k$ by equation (5.1) and wish to compare our result with that obtained in [2] for an isotropic elastic material with Poisson's ratio $\sigma=0 .^{3}$ ) In [2] an exponential decay law of the type (4.28) was established with decay constant $k_{0}>0$ given by ${ }^{4}$ )

$$
k_{0}^{2}=\frac{\pi^{2}}{2 b^{2}}(\sqrt{2}-1) \text {. }
$$

In the present case, $k$ is determined from (5.1), which reads

$$
k^{2}=\frac{\pi^{2}}{2 b^{2}} \frac{\lambda s}{\beta_{22}},
$$

where $\lambda>0$ is the minimum eigenvalue of the matrix

$$
B=\left[\begin{array}{lll}
\beta_{11} & \beta_{12} & \frac{\beta_{16}}{\sqrt{2}} \\
\beta_{12} & \beta_{22} & \frac{\beta_{26}}{\sqrt{2}} \\
\frac{\beta_{16}}{\sqrt{2}} & \frac{\beta_{26}}{\sqrt{2}} & \frac{\beta_{66}}{2}
\end{array}\right] .
$$

We note here that for an orthotropic material (with the coordinate planes as planes of elastic symmetry) we have $\beta_{26}=0, \beta_{16}=0$ while for an isotropic material we obtain in addition $\beta_{11}=\beta_{22}=(1-\sigma) / 2 \mu, \beta_{66}=1 / \mu, \beta_{12}=-\sigma / \mu$, where $\mu$ is the shear modulus.

While $\lambda$ could be determined explicitly for a given anisotropic material, for our purposes it is sufficient to use the following upper bound result from matrix analysis. For any real symmetric matrix $B$, we have

$$
\lambda \leqq \min \left(\beta_{11}, \beta_{22}, \frac{\beta_{66}}{2}\right) \text {, }
$$

1) See Uspensky [22], Ch. VI, for example.

2) The value $s_{2}$ could be calculated from the roots of a quartic given in [22]. We require merely the result (5.9).

${ }^{3}$ ) The stresses in [2] are independent of Poisson's ratio; consequently only the case $\sigma=0$ required consideration in that reference.

$\left.{ }^{4}\right)$ See (3.4), (3.21) of [2]. 
or, using the notation introduced in (5.2), we obtain

$$
\frac{\lambda}{\beta_{22}} \leqq \min \left(\frac{\beta_{11}}{\beta_{22}}, 1, \alpha\right) \text {. }
$$

For a discussion of the bound (5.13), see $\S 11$ Chapter 8 of Bellman [23] or Taussky [24].

In Case 1, we now show, using obvious notation, that

$$
\frac{\lambda_{1}}{\beta_{22}} s_{1} \leqq \sqrt{2}-1 \text {, }
$$

and so we may conclude from $(5.10),(5.11)$ that

$$
k_{1} \leqq k_{0} \text {. }
$$

The function $\left(\alpha^{2}+1\right)^{\frac{1}{2}}-\alpha$ is a monotonic decreasing function of $\alpha$ for all $\alpha>0$ and so for $\alpha \geqq 1, s_{1}=\left(\alpha^{2}+1\right)^{\frac{1}{2}}-\alpha \leqq \sqrt{2}-1$, with equality holding when $\alpha=1$. Furthermore, when $\alpha \geqq 1,(5.14)$ implies that $\left(\lambda_{1} / \beta_{22}\right) \leqq 1$; if $\alpha=1$, it can be readily shown that $\left(\lambda_{1} / \beta_{22}\right)=1$ if and only if $B$ is strictly diagonal. $\left.{ }^{1}\right)$ Thus, when $\alpha \geqq 1,(5.15)$ clearly holds, with equality in the limiting case mentioned. When $\alpha<1,(5.14)$ yields $\left(\lambda_{1} / \beta_{22}\right) \leqq \alpha$ and from (5.8) we find that

$$
\frac{\lambda_{1}}{\beta_{22}} s_{1} \leqq \alpha\left[\left(\alpha^{2}+1\right)^{\frac{1}{2}}-\alpha\right]<\sqrt{2}-1 \text {, }
$$

the final step arising from the monotonic increasing character of the function $\alpha\left[\left(\alpha^{2}+1\right)^{\frac{1}{2}}-\alpha\right]$ for $\alpha>0$. In Case 2, the inequality (5.14) is still valid and using the result (5.9), the above argument may be used to show that

$$
k_{2}<k_{0} \text {. }
$$

Thus we have established in (5.16), (5.18) that the decay constant furnished by the present analysis for the anisotropic plane problem is less than that obtained in [2] for the isotropic case. Since these decay constants are lower bounds on the actual rate of decay, this result does not preclude the possibility that the relationship between the actual rates is reversed; however our results suggest the conjecture that the actual rate of decay in the isotropic case is the greater, or in other words, that the "characteristic decay length" associated with Saint-Venant's principle in plane elastostatics is smallest in the isotropic case. The foregoing remarks indicate that caution must be observed in invoking traditional Saint-Venant principle type arguments in elasticity problems involving anisotropic media.

\section{Acknowledgment}

The author wishes to express his appreciation to Professor J. K. Knowles of California Institute of Technology under whose guidance this work was initiated and who offered many helpful suggestions for improving the manuscript.

\section{REFERENCES}

[1] Toupin, R. A., Saint-Venant's principle. Archive for Rational Mechanics and Analysis 18 (1965) 83-96.

[2] Knowles, J. K., On Saint-Venant's principle in the two-dimensional linear theory of elasticity. Archive for Rational Mechanics and Analysis 21 (1966) 1-22.

1) This corresponds to the isotropic case when $\sigma=0$ 
[3] Knowles, J. K. and E. Sternberg, On Saint-Venant's principle and the torsion of solids of revolution. Archive for Rational Mechanics and Analysis 22 (1966) 100-120.

[4] Knowles, J. K. and C. O. Horgan, On the exponential decay of stresses in circular elastic cylinders subject to axisymmetric self-equilibrated end loads. International Journal of Solids and Structures 5 (1969) 33-50.

[5] Roseman, J. J., A pointwise estimate for the stress in a cylinder and its application to Saint-Venant's principle. Archive for Rational Mechanics and Analysis 21 (1966) 23-48.

[6] Roseman, J. J., The principle of Saint-Venant in linear and non-linear plane elasticity. Archive for Rational Mechanics and Analysis 26 (1967) 142-162.

[7] Knowles, J. K., A Saint-Venant principle for a class of second-order elliptic boundary value problems. Zeitschrift für angewandte Mathematik und Physik (ZAMP) 18 (1967) 473-490.

[8] Wu, C-H., A Saint-Venant principle for the Neumann problem with a non-thin two-dimensional domain. International Journal of Engineering Science 8 (1970) 389-402.

[9] Edelstein, W. S., On Saint-Venant's principle in linear viscoelasticity. Archive for Rational Mechanics and Analysis 36 (1970) 366-380.

[10] Edelstein, W. S., A spatial decay estimate for the heat equation. Zeitschrift für angewandte Mathematik und Physik (ZAMP) 20 (1969) 900-905.

[11] Edelstein, W. S., Further study of spatial decay estimates for semilinear parabolic equations. Journal of Mathematical Analysis and Applications 35 (1971) 577-590.

[12] Sigillito, V. G., On the spatial decay of solutions of parabolic equations. Zeitschrift für angewandte Mathematik und Physik (ZAMP) 21 (1970) 1078--1081.

[13] Knowles, J. K., On the spatial decay of solutions of the heat equation. Zeitschrift für angewandte Mathematik und Physik (ZAMP) 22 (1971) 1050-1056.

[14] Sternberg, E., On Saint-Venant's principle. Quarterly of Applied Mathematics 11 (1954) 393-402.

[15] Sternberg, E., On some recent developments in the linear theory of elasticity, in Structural Mechanics, 48-73. (Proceedings of First Symposium on Naval Structural Mechanics, 1958. Edited by J. N. Goodier and N. J. Hoff.) Pergamon Press, New York (1960).

[16] Lekhnitskii, S. G., Theory of Elasticity of an Anisotropic Elastic Body. (Translated by P. Fern.) Holden-Day, San Francisco (1963).

[17] Hardy, G. H., J. E. Littlewood, and G. Pólya, Inequalities. University Press, Cambridge (1959).

[18] Courant, R., and D. Hilbert, Methods of Mathematical Physics, Volume I. Interscience, New York (1953).

[19] Gould, S. H., Variational Methods for Eigenvalue Problems. University of Toronto Press, Toronto (1957).

[20] Pini, B., Sulle equazioni lineari a derivate parziali d'ordine 2 di tipo ellittico e sui sistemi ellittici di equazioni lineari del secondo ordine sopra una superficie chiusa. Rendiconti di Matematica e delle sue Applicazioni (Università di Roma) Serie 5, 11 (1952) 176-195.

[21] Miranda, C., Partial Differential Equations of Elliptic Type. Second Revised Edition. (Translated by Z. C. Motteler.) Springer-Verlag, Berlin (1970).

[22] Uspensky, J. V., Theory of Equations. McGraw-Hill, New York (1948).

[23] Bellman, R., Introduction to Matrix Analysis. McGraw-Hill, New York (1960).

[24] Taussky, O., Some topics concerning bounds for eigenvalues of finite matrices, in Survey of Numerical Analysis, 279-297. (Edited by J. Todd) McGraw-Hill, New York (1962).

\section{Note added in proof:}

In a forthcoming paper in this journal [1] the above analysis is applied to the special case of a transversely isotropic material with a high degree of anisotropy. Disagreement with SaintVenant's principle is anticipated, confirming recent observations made by other authors [2], [3] in similar contexts.

\section{REFERENCES}

[1] Horgan, C. O., Some remarks on Saint-Venant's principle for transversely isotropic composites. Journal of Elasticity (forthcoming).

[2] Everstine, G. C. and A. C. Pipkin, Stress channelling in transversely isotropic elastic composites. Zeitschrift für angewandte Mathematik und Physik (ZAMP) 22 (1971) 825-834.

[3] Rogers, T. G. and A. C. Pipkin, Small deflections of fiber-reinforced beams or slabs. Journal of Applied Mechanics 38 (1971) 1047-1048. 